\title{
Protein tyrosine phosphatase 1B negatively regulates S100A9-mediated lung damage during respiratory syncytial virus exacerbations
}

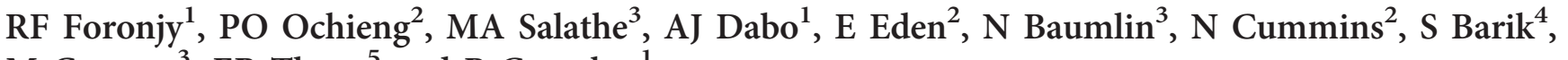 \\ M Campos ${ }^{3}$, EB Thorp ${ }^{5}$ and P Geraghty ${ }^{1}$
}

Protein tyrosine phosphatase 1B (PTP1B) has anti-inflammatory potential but PTP1B responses are desensitized in the lung by prolonged cigarette smoke exposure. Here we investigate whether PTP1B expression affects lung disease severity during respiratory syncytial viral (RSV) exacerbations of chronic obstructive pulmonary disease (COPD). $P t p 1 b^{-1-}$ mice infected with RSV exhibit exaggerated immune cell infiltration, damaged epithelial cell barriers, cytokine production, and increased apoptosis. Elevated expression of S100A9, a damage-associated molecular pattern molecule, was observed in the lungs of $P t p 1 b^{-1-}$ mice during RSV infection. Utilizing a neutralizing anti-S100A9 IgG antibody, it was determined that extracellular S100A9 signaling significantly affects lung damage during RSV infection. Preexposure to cigarette smoke desensitized PTP1B activity that coincided with enhanced S100A9 secretion and inflammation in wild-type animals during RSV infection. S100A9 levels in human bronchoalveolar lavage fluid had an inverse relationship with lung function in healthy subjects, smokers, and COPD subjects. Fully differentiated human bronchial epithelial cells isolated from COPD donors cultured at the air liquid interface secreted more S100A9 than cells from healthy donors or smokers following RSV infection. Together, these findings show that reduced PTP1B responses contribute to disease symptoms in part by enhancing \$100A9 expression during viral-associated COPD exacerbations.

\section{INTRODUCTION}

Protein tyrosine phosphatase 1B (PTP1B) is primarily known to negatively regulate insulin receptor signaling, with Ptp $1 b^{-1-}$ mice displaying hypersensitivity to insulin. ${ }^{1}$ PTP1B regulates several other receptors, such as the Ephrin receptor, Toll-like receptor (TLR) signaling, and leptin receptor signaling. ${ }^{2}$ In addition to these receptors, PTP1B has multiple other substrates, including $\mathrm{c}-\mathrm{Src}$, protein phosphatase $2 \mathrm{~A}$, and $\mathrm{Bcr}-$ $\mathrm{Abl}^{2}$ Though PTP1B is the most studied tyrosine phosphatase, its role in the lung is not well defined, especially in infection models. PTP1B expression can influence cell recruitment and expansion, with $P t p 1 b^{-1-}$ mice having increased B-cell numbers in the bone marrow and lymph nodes, ${ }^{3}$ and increased leukocytes in the lungs in an animal asthma model. ${ }^{4}$ In addition, the loss of $P t p 1 b$ in mice compromises macrophage viability. ${ }^{5}$ Our group has determined that enhancing PTP1B expression in cigarette smoke conditions can resolve inflammation and protease production in the lungs. ${ }^{6}$ As PTP1B regulates immune cell responses and is altered during lung diseases, investigating the impact of PTP1B deficiency during viral infection is of great importance.

Chronic obstructive pulmonary disease (COPD) is the third leading cause of death in the United States, ${ }^{7}$ and the majority of COPD subjects experience at least one exacerbation per year that results in a substantial number of hospital admissions. ${ }^{8}$ Despite improving detection methods utilized to identify pulmonary pathogens in hospitalized patients, the majority of infections are not detectable in adults with community-

${ }^{1}$ Division of Pulmonary and Critical Care Medicine, SUNY Downstate Medical Center, Brooklyn, New York, USA. ${ }^{2}$ Division of Pulmonary and Critical Care Medicine, Mount Sinai Roosevelt, Mount Sinai Health System, New York, New York, USA. ${ }^{3}$ Division of Pulmonary, Allergy, Critical Care, and Sleep Medicine, University of Miami, Miami, Florida, USA. ${ }^{4}$ Center for Gene Regulation in Health and Disease, and Department of Biological, Geological and Environmental Sciences, Cleveland State University, Cleveland, Ohio, USA and ${ }^{5}$ Department of Pathology and Feinberg Cardiovascular Research Institute, Feinberg School of Medicine, Northwestern University, Chicago, Illinois, USA. Correspondence: P Geraghty (Patrick.Geraghty@downstate.edu) 
acquired pneumonia and viruses are detected more frequently than bacteria. ${ }^{9}$ Thus, viral infections are considered a major driving factor of COPD exacerbations and thus contribute to disease morbidity and mortality. Rhinovirus, influenza, and respiratory syncytial virus (RSV) are frequently detected in the respiratory tract of COPD patients during an exacerbation. ${ }^{10}$ Despite the high frequency of RSV detected during a COPD exacerbation, ${ }^{11}$ few studies have examined the pathogenicity of RSV in COPD animal models. ${ }^{12,13}$ Patients infected with RSV, usually infants, the elderly, and immunocompromised patients and also healthy adults, ${ }^{14}$ develop mild to severe symptoms, including fever, mucus production, and wheezing. RSV infection triggers several pathogen-associated molecular pattern (PAMP) receptors that are regulated by PTP1B. ${ }^{15,16}$ However, the ability of RSV to elicit a damage-associated molecular pattern (DAMP) response and the potential regulation of DAMPs by PTP1B are not known. DAMPs are produced from infected, damaged, or dead cells and induce a potent inflammatory response ${ }^{17}$ that could enhance the severity of a COPD exacerbation.

In this study, we determined that a DAMP protein, S100A9, is negatively regulated by PTP1B and loss of PTP1B expression enhances S100A9 expression and worsens lung injury during RSV infection. S100A9 protein induced potent inflammatory responses and enhances lung cell death during RSV infection. Bronchoalveolar lavage fluid (BALF) from healthy human subjects, smokers, and COPD patients showed an inverse related relation of S100A9 levels with lung function. Utilizing an in vitro model of viral exacerbations in primary airway epithelial cells from patients, we have demonstrated that cells from COPD patients have reduced PTP1B activity, coinciding with heightened S100A9 secretion. The activation of DAMP responses contributes to viral clearance. ${ }^{18}$ However, unregulated and sustained DAMP signaling could play a part in lung disease exacerbations that enhance the loss of lung function. Therefore, maintaining an effective lung PTP1B response aids in minimizing lung damage induced by S100A9 expression.

\section{RESULTS}

\section{Cigarette smoke exposure desensitizes lung PTP1B responses and deficiency of PTP1B expression enhances susceptibility to cigarette smoke in mice}

We have previously observed that PTP1B counters lung inflammation ${ }^{6}$ and reduced PTP1B lung activity is observed during RSV infection. ${ }^{12}$ To investigate the effect of acute and chronic cigarette smoke exposure on PTP1B activity, FVB/NJ mice were exposed to daily cigarette smoke exposure for 2 weeks (acute) or 6 months (chronic). Acute smoke exposure resulted in a robust PTP1B response in the lungs that was desensitized following chronic smoke exposure (Figure 1a). Further studies were performed to determine how the loss of PTP1B affects lungs during smoke exposure. Ptp $1 b^{-1-}$ mice and their wild-type littermates were exposed to 4 months of smoke and immune cell infiltration and lung remodeling were examined. Loss of Ptp $1 b$ expression in mice increased BALF immune cell infiltration (Figure 1b) and lung remodeling, as a

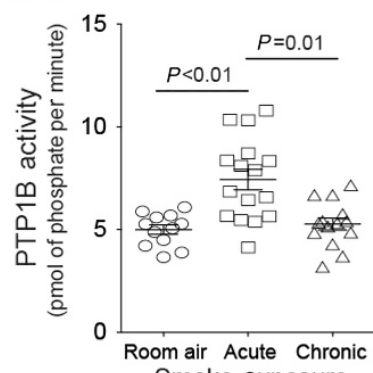

b $\square$ Room air $\square$ Cigarette smoke

Smoke exposure
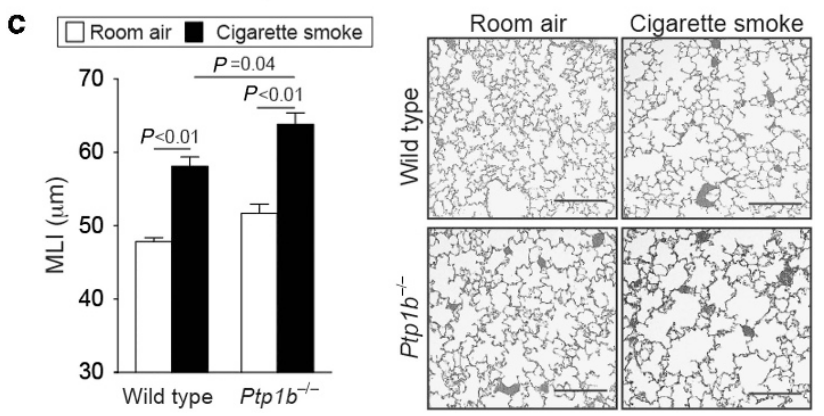

Figure 1 Loss of protein tyrosine phosphatase 1B (PTP1B) expression enhances lung remodeling. (a) Enhanced PTP1B activity is observed in the lungs of $F V B / N J$ mice exposed to 2 weeks (acute) of cigarette smoke exposure but not following 6 months (chronic) of exposure. (b) Ptp $1 b^{-1-}$ mice and their FVB/NJ wild-type littermates were exposed to 4 months of cigarette smoke or room air. Bronchoalveolar lavage fluid (BALF) immune cellularity was enhanced by smoke exposure and was further enhanced by loss of Ptp $1 b$ expression. (c) Mean linear intercepts (MLIs) were measured in the lungs of the mice to assess lung remodeling and comparative histology images of the four mouse groups are presented here (scale bar $=40 \mu \mathrm{m}$ ). Slides were randomized, read blindly, and scored for MLI. Graphs are represented as mean \pm s.e.m., where $n$ is at least 10 animals per group for each time point. The $P$ values are shown, comparing both groups connected by a line.

determined by mean linear intercept analysis (Figure 1c). These results establish that the loss of PTP1B enhanced the susceptibility to smoke-induced lung damage. Thus, the desensitizing of PTP1B activity by cigarette smoke exposure could be a major contributory factor to disease progression.

\section{Loss of Ptp1b expression results in increased lung damage during RSV infection in mice}

$P t p 1 b^{-1-}$ mice and their wild-type littermates were infected with RSV. For the first 3 days post infection (DPI), $P t p 1 b^{-1-}$ and wild-type mice had comparable immune cell infiltration. As the infection progressed, $P t p 1 b^{-1-}$ mice had elevated macrophage, neutrophil, lymphocyte, and eosinophil infiltration into their lungs and airways compared with wild-type animals (Figure 2a). RSV-infected mice exhibited perivascular lymphocytic inflammation and increased inflammatory cell infiltration around the bronchial airways and within the alveolar spaces that was significantly elevated in $P t p 1 b^{-1-}$ mice at 7 DPI as compared with wild-type mice (Figure $2 \mathbf{b}$ ). RSV readily infected the airway epithelium in large and small airways, determined by the detection of RSV antigen in both mouse groups (Figure 2c). $P t p 1 b^{-1-}$ and wild-type mice had 
a $\Theta$ Wild type + Mock $\triangle P \operatorname{tp} 1 b^{-1-}+$ Mock $\bullet$ Wild type + RSV $\neq P \operatorname{tp} 1 b^{-i-}+\mathrm{RSV}$
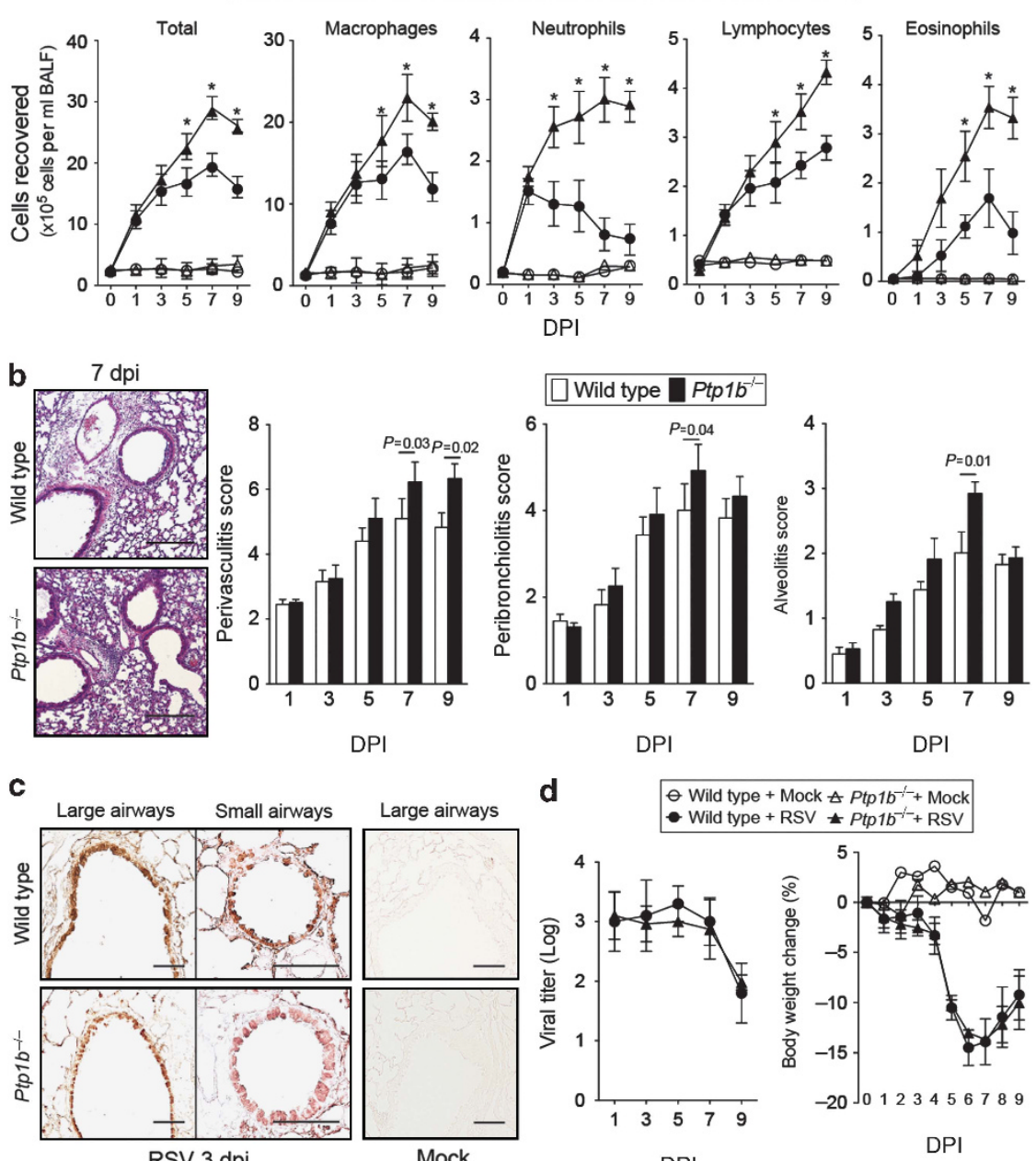

Figure 2 Ptp1b-deficient mice display enhanced lung immune cell infiltration during respiratory syncytial virus (RSV) infection. (a) Ptp $1 b^{-1-}$ mice (triangle) and their FVB/NJ wild-type littermates (circle) were infected with $1 \times 10^{6}$ plaque-forming units (PFUs) of RSV (closed) or mock control (open) and animals were killed on $0,1,3,5,7$, and 9 days post infection (DPI). Bronchoalveolar lavage fluid (BALF) immune cellularity was determined for total immune cell number, macrophages, neutrophils, lymphocytes, and eosinophils. ${ }^{*} P<0.05$ comparing $P$ tp $1 b^{-1-}$ mice with wild-type mice 9 days after RSV challenge. (b) Comparative histology images of infected animals from each background at 7 DPI are presented here (scale bar $=50 \mu \mathrm{m})$.

Histopathology (peribronchiolitis, perivasculitis, and alveolitis scoring) was recorded in mice for each group. Slides were randomized, read blindly, and scored for each parameter. The $P$ values are shown, comparing both treatments connected by a line. (c) Immunohistochemistry was performed on lung tissue from both mouse groups at 3 DPI with an antibody that recognizes RSV antigen (brown). Mock-treated animals demonstrate negative staining for RSV antigen. (d) RSV infectivity and animal body weight was comparable in wild-type and Ptp $1 b^{-1-}$ mice. Each graph is represented as mean \pm s.e.m. Each measurement was performed on 10 animals per group, with 6 replicates per animal. PTP1B, protein tyrosine phosphatase 1B.

comparable RSV titer and weight changes during infection (Figure 2d). Therefore, loss of Ptp1b expression during RSV infection leads to exaggerated inflammation, without affecting viral infectivity.

Simultaneously, $P t p 1 b^{-1-}$ mice had significantly higher protein concentration (Figure 3a) in the BALF compared with infected wild-type mice, suggesting potentially increased damage and disruption of the epithelial cell barrier. Enhanced apoptosis and caspase-3 cleavage were observed in the $P t p 1 b^{-1-}$ mice at 5 DPI (Figure 3a,b). Furthermore, PTP1B expression negatively regulated the expression of RANTES (regulated on activation, normal $\mathrm{T}$ cell expressed and secreted), PDGF-bb (platelet-derived growth factor-bb),
G-CSF (granulocyte colony-stimulating factor), CXCL1, CXCL9, CXCL10, MCP-1 (monocyte chemoattractant protein-1), Eotaxin, interleukin (IL-4), IL-5, and IL-13, observed at 5 DPI (Figure 3c). These inflammation changes may have been influenced by enhanced expression of MyD88 (myeloid differentiation primary response gene 88 ) and TRIF (TIR-domain-containing adapter-inducing interferon- $\beta$ ) and the phosphorylation of IRF3 (interferon regulatory factor 3), IRF7 (interferon regulatory factor 7) and TBK1 and activation of nuclear factor- $\mathrm{\kappa B}$ during infection in $P t p 1 b$ deficiency compared with wild-type mice (Supplementary Figure S1 online). Therefore, PTP1B regulates cytokine signaling associated with lung damage. 
a

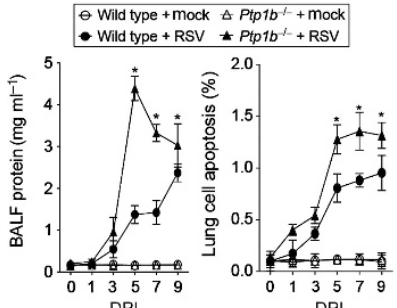

C

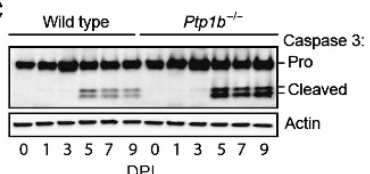

b
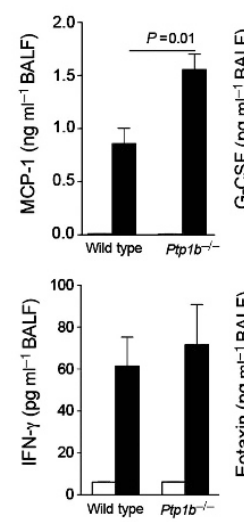
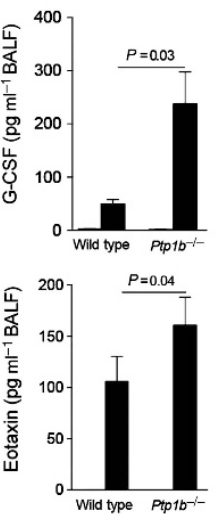
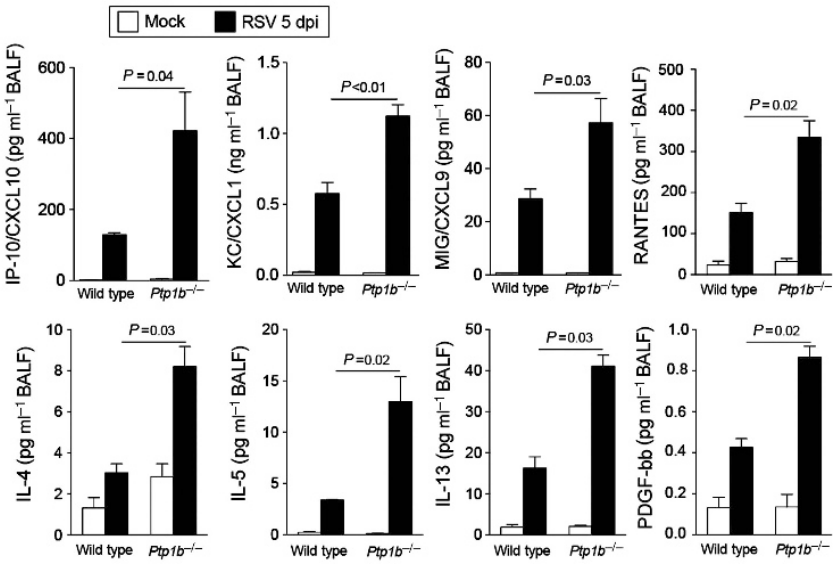

Figure 3 Ptp $1 b$-deficient mice display enhanced lung damage and cytokines during respiratory syncytial virus (RSV) infection. (a) Ptp $1 b^{-1-}$ mice (triangle) and their FVB/NJ wild-type littermates (circle) were infected with $1 \times 10^{6}$ plaque-forming units (PFUs) of RSV (closed) or mock control (open) and animals were killed on $0,1,3,5,7$, and 9 days post infection (DPI). Ptp $1 b^{-1-}$ mice have enhanced protein content in bronchoalveolar lavage fluid (BALF) compared with wild-type mice. $P t p 1 b^{-1-}$ mice display greater lung apoptosis. ${ }^{*} P<0.05$ compared with wild-type mice on each corresponding time point. (b) Loss of PTP1B expression enhances caspase-3 cleavage and activation as determined by caspase-3 immunoblots conducted on lung tissue lysates. (c) BALF levels of MCP-1, G-CSF, CXCL10, CXCL1, CXCL9, PDGF-bb, RANTES, IFN- $\gamma$, Eotaxin, IL-4, IL-5, and IL-13 were determined in both mouse genotypes following $5 \mathrm{DPI}$. Graphs are represented as mean \pm s.e.m., where $n$ is at least 10 per group for each time point. $P$ values are shown, comparing both treatments connected by a line. CXCL1, chemokine (C-X-C motif) ligand 1; CXCL9, chemokine (C-X-C motif) ligand 9; CXCL10, chemokine (C-X-C motif) ligand 10; G-CSF, granulocyte colony-stimulating factor; IFN- $\gamma$, interferon- $\gamma$; IL, interleukin; MCP-1, monocyte chemoattractant protein-1; PDGF-bb, platelet-derived growth factor-bb; PTP1B, protein tyrosine phosphatase 1B; RANTES, regulated on activation, normal T cell expressed and secreted.

\section{PTP1B suppresses S100A9 expression during RSV infection}

Quantitative PCR (qPCR) arrays were performed to investigate the key Ptp1b-regulated genes during RSV infection that contribute to enhanced apoptosis. Over 350 genes associated with cell death were screened and 24 of these genes were regulated by Ptp 1 b expression (Figure 4a,b). One of these 24 genes, S100A9, was further investigated as S100A9 regulates inflammation and disease progression during an animal influenza infection model ${ }^{17}$ and is also regulated by TRIF $^{17}$ and can activate TLR4. ${ }^{19}$ PTP1B can directly affect these signaling processes. ${ }^{15,16}$ In humans, S100A9 levels are elevated in the serum of COPD patients during an exacerbation. ${ }^{20}$ We detected enhanced S100A9 levels in the BALF (Figure 4c) and increased S100A9 in lung tissue (Figure 4d) during RSV infection in mice. Lung tissue was examined at 5 DPI as animals had significantly heightened inflammation (Figure 3c), apoptosis (Figure 3b), and maximum viral load (Figure 2d) in their lungs on this day. The induction of S100A9 during RSV infection was further confirmed in human primary small airway epithelial (SAE) cells and mouse bone marrow-derived macrophages (Figure 4e). Airway epithelial cells were utilized, as this was the primary region of the lungs infected by RSV in the mouse model (Figure 2c). Interestingly, stimulation with S100A9 protein induces enhanced gene expression of PTP1B-negatively regulated cytokines in SAE cells (Figure 4f). Indeed, silencing of PTP1B expression (see silencing small interfering RNA (siRNA) efficiency in Supplementary Figure S2A) enhances the expression of MCP-1, PDGF-bb, CXCL10, CXCL1, and CXCL9 following S100A9 stimulation (Figure 4f). Therefore, S100A9 signaling could influence lung inflammation during RSV infection.

\section{Extracellular S100A9 regulates inflammation and apoptosis during RSV infection}

As extracellular S100A9 induces similar inflammatory responses compared with $P T P 1 B$ deficiency in SAE cells, we hypothesized that extracellular S100A9 significantly contributes to the lung inflammation observed in $P t p 1 b^{-/-}$mice. To investigate this, we used an anti-S100A9 blocking antibody that neutralizes extracellular S100A9 signaling. At $2 \mathrm{~h}$ before RSV infection, animals were intraperitoneally administered either anti-S100A9 immunoglobulin G (IgG) or isotype control IgG, as depicted in Figure 5a. $P t p 1 b^{-/-}$and wild-type mice that received anti-S100A9 IgG had little to no weight changes during infection unlike animals that received control IgG (Figure 5b). Blocking extracellular S100A9 signaling also subdued immune cell infiltration into the lungs (Figure 5c) and perivascular lymphocytic inflammation, inflammatory cell infiltration around the bronchial airways, and within the alveolar spaces of both animal backgrounds at 5 DPI (Figure 5d).

Subduing S100A9 signaling also diminished protein concentration in the BALF compared with control IgG-treated animals (Figure 6a) and reduced RSV infection-induced apoptosis (Figure 6b). Diminished cleavage of caspase-3 was observed in animals treated with the S100A9 neutralizing antibody compared with IgG-treated animals (Supplementary Figure S3). Blocking extracellular S100A9 signaling had little to no effect on RSV infectivity (Figure 6c) but significantly decreased MCP-1, Eotaxin, IL-4, IL-5, and IL-13 release in both wild-type and $P t p 1 b^{-1-}$ animals that received antiS100A9 IgG (Figure 6d). These results demonstrated that PTP1B-regulated S100A9 contributes to the severity of lung inflammation and apoptosis during RSV infection. 
a

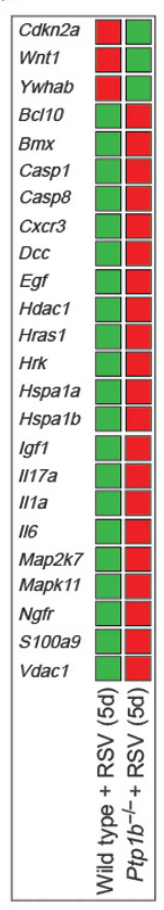

b
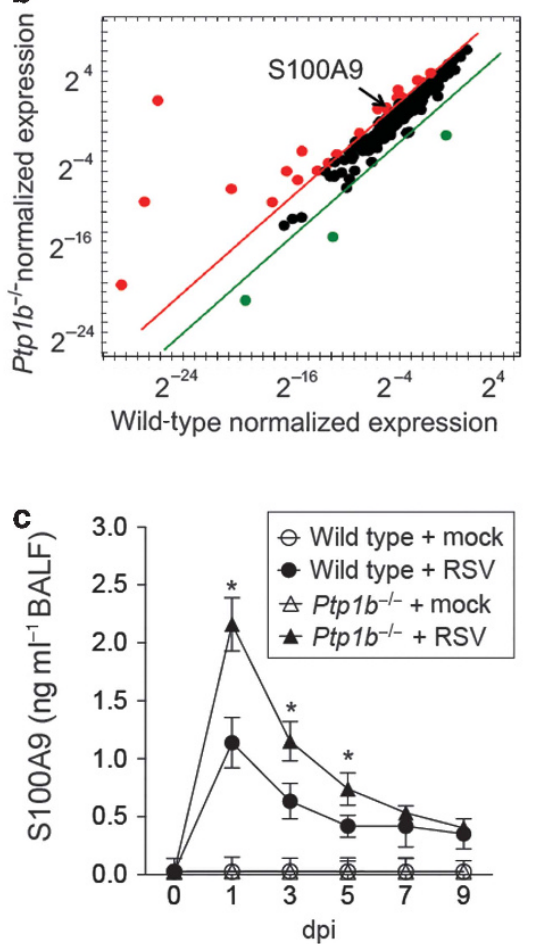

d
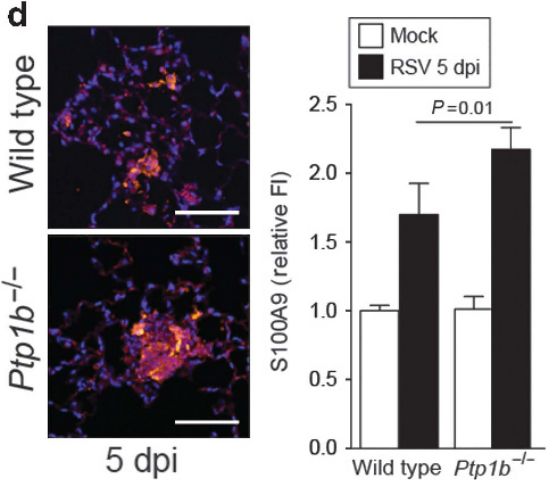

Wild-type

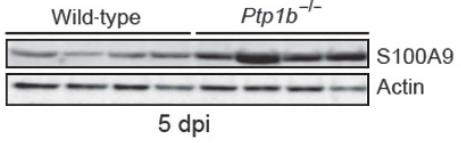

e Human SAE cells
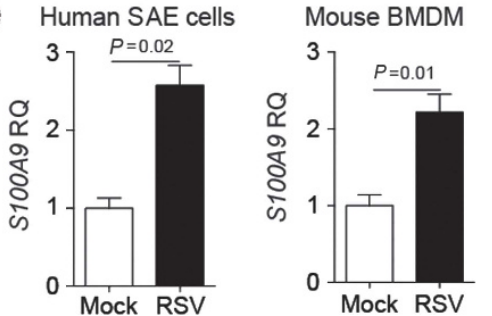

f

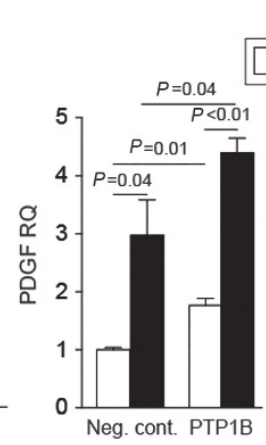

Human SAE cells
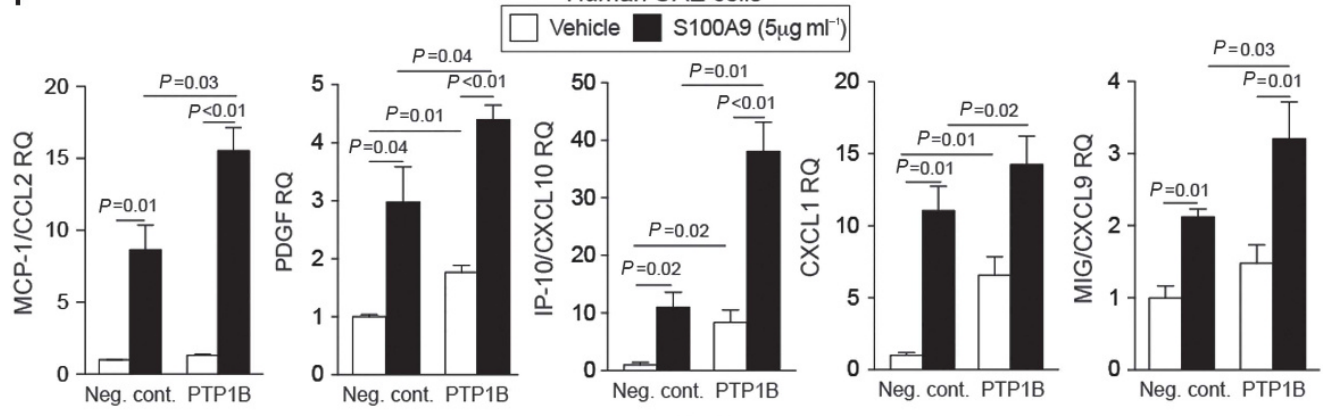

Figure 4 Protein tyrosine phosphatase 1B (PTP1B) hinders S100A9 expression during respiratory syncytial virus (RSV) infection. Gene expression profiling of lung tissue from wild-type and Ptp1b-deficient mice was performed 5 days post infection (DPI). Quantitative PCR (qPCR) arrays were performed using the Bio-Rad PrimePCR Apoptosis and Survival panel to examine over 350 genes associated with cell survival and apoptosis. (a) Data were analyzed with the Bio-Rad PrimePCR analysis software. Color code in the clustergram shows standardized gene expression responses from the microarray probes of samples from RSV-infected wild-type and $P t p 1 b^{-1-}$ mice. Red indicates higher expression and green lower expression. $5 \mathrm{~d}, 5 \mathrm{days}$. (b) Expression values were $\log _{2}$ transformed and normalized to compare wild-type and $P \operatorname{tp} 1 b^{-1-}$ gene expression. Threshold lines demonstrate significantly greater differences in expression between both groups. S100A9, a gene negatively regulated by PTP1B, was examined in the (c) bronchoalveolar lavage fluid (BALF) and (d) lung tissue of wild-type and Ptp $1 b^{-1-}$ mice. (d) Tissue was stained for RSV antigen (red) and fluorescent intensity (FI) quantified. S100A9 immunoblots were also conducted on lung tissue. (e) Gene expression of S100A9 is inducible in human small airway epithelial (SAE) cells and murine bone marrow-derived macrophages (BMDMs) following RSV infection. (f) Stimulus of S100A9 protein induces release of MCP-1, PDGF, CXCL10, CXCL1, and CXCL9 from SAE human cells transfected with negative (Neg.)/scrambled or PTP1B small interfering RNA (siRNA). Graphs are represented as mean \pm s.e.m., where $n=10$ per group. qPCR is represented as relative quantification $(\mathrm{RQ})$. (c) ${ }^{\star} P<0.05 \mathrm{compared}$ with wild-type mice on each corresponding time point. (e, f) $P$ values are shown, comparing both treatments connected by a line. CXCL1, chemokine (C-XC motif) ligand 1; CXCL9, chemokine (C-X-C motif) ligand 9; CXCL10, chemokine (C-X-C motif) ligand 10; MCP-1, monocyte chemoattractant protein-1; PDGF, platelet-derived growth factor.

\section{Preexposure to cigarette smoke enhances S100A9 expression during an RSV infection}

Here we examined whether chronic smoke exposure before RSV infection would affect S100A9-associated inflammation and apoptosis in the lungs. Animals were exposed to either room air or cigarette smoke for $4 \mathrm{~h}$ daily for 4 months before
RSV infection as depicted in Figure 7a. Elevated viral titers were observed in smoke exposed animals from both wild-type and $P t p 1 b^{-1-}$ mice at 9 DPI as compared with room airtreated mice (Figure $7 \mathbf{b}$ ). Exposure to 4 months of smoke before RSV infection desensitized PTP1B activity further in wild-type mice at 9 DPI (Figure 7c). Unlike animals exposed to 
a

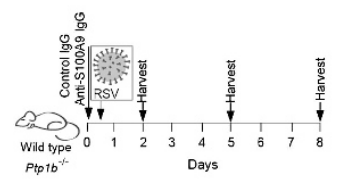

C
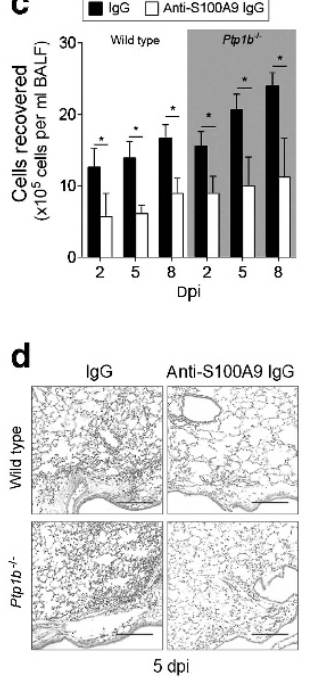
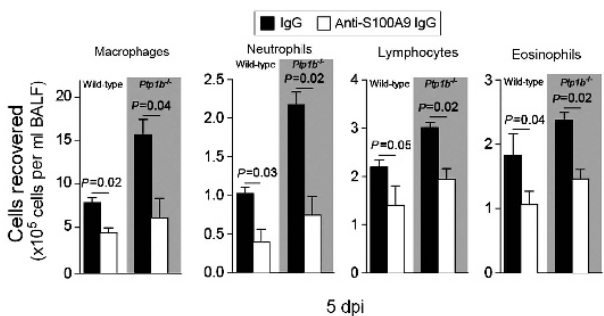

$5 \mathrm{dpi}$

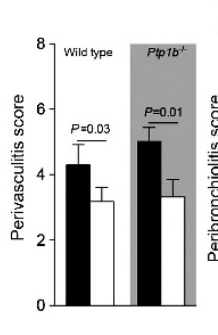

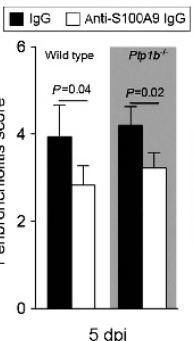

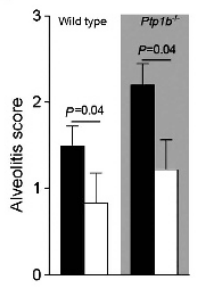

Figure 5 Infiltration of immune cells following respiratory syncytial virus (RSV) infection is diminished upon neutralization of extracellular S100A9 signaling. (a) Wild-type and Ptp $1 b^{-1-}$ mice were intraperitoneally (IP) injected with isotype control immunoglobulin G (IgG) or anti-S100A9 IgG before intranasal infection with $1 \times 10^{6}$ plaque-forming units (PFUs) of RSV and animals were killed 2, 5, and 8 days post infection (DPI). (b) Animal body weight and (c) bronchoalveolar lavage fluid (BALF) cellularity (total BAL cells, macrophages, neutrophils, lymphocytes, and eosinophils) were determined in each group (white background $=$ wild-type and gray area $=P t p 1 b^{-1-}$ mice). (d) Comparative histology images of infected animals at $5 \mathrm{DPI}$ are presented here (scale bar $=50 \mu \mathrm{m}$ ). Histopathology (peribronchiolitis, perivasculitis, and alveolitis scoring) was recorded in mice for each group. Slides were randomized, read blindly, and scored for each parameter. Graphs are represented as mean \pm s.e.m., where each measurement was performed 3 times on 10 animals per group. ${ }^{\star} P<0.05$ or $P$ values are shown, comparing both treatments connected by a line.

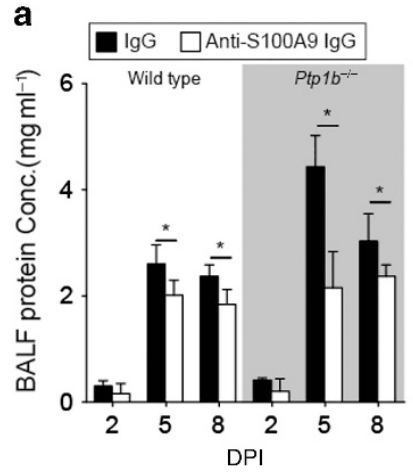

d
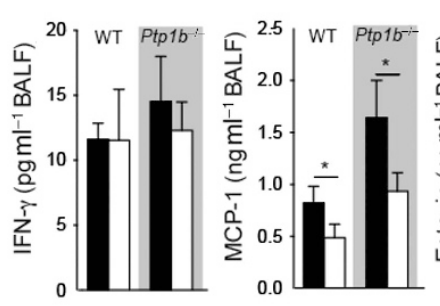

b

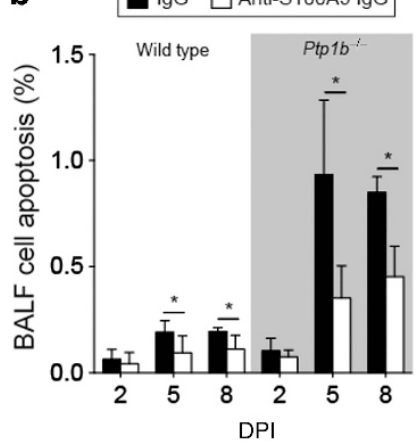

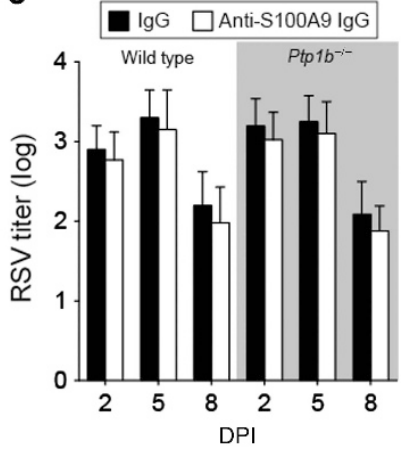

IgG $\square$ Anti-S100A9 IgG
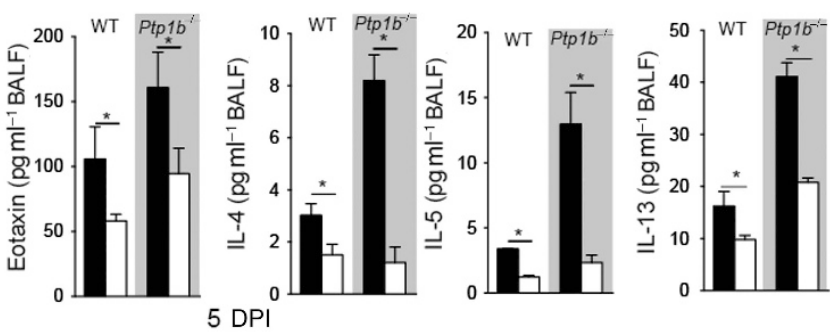

Figure 6 Extracellular S100A9 signaling neutralization reduces lung damage and cytokine production during respiratory syncytial virus (RSV) infection. Wild-type and $P t p 1 b^{-1-}$ mice were intraperitoneally (IP) injected with isotype control immunoglobulin G (IgG) or anti-S100A9 IgG before intranasal infection with $1 \times 10^{6}$ plaque-forming units (PFUs) of RSV and animals were killed 2, 5, and 8 days post infection (DPI). (a) Bronchoalveolar lavage fluid (BALF) protein content, (b) BALF cells undergoing apoptosis, and (c) lung viral titer were determined in each group (white background = wild-type and gray area $=$ Ptp $1 b^{-1-}$ mice). (d) Monocyte chemoattractant protein-1 (MCP)-1/CCL2, interferon- $\gamma($ IFN- $\gamma$ ), Eotaxin, interleukin (IL)-4, IL-5, and IL-13 levels were determined in BALF by luminex assays at $5 \mathrm{DPI}$. Graphs are represented as mean \pm s.e.m., where each measurement was performed on 10 animals per group, with 6 replicates per animal. ${ }^{*} P<0.05$, comparing both treatments connected by a line. Conc, concentration; PTP1B, protein tyrosine phosphatase 1B; WT, wild type.

room air, BALF S100A9 levels were similarly increased in smoke-exposed wild-type and $P t p 1 b^{-/-}$animals during RSV infection (Figure 7d). Similarly, immune cell infiltration and protein concentration in the BALF were comparably increased in wild-type and $P t p 1 b^{-1-}$ mice during RSV infection following smoke exposure (Figure 7e,f). In contrast, 
a
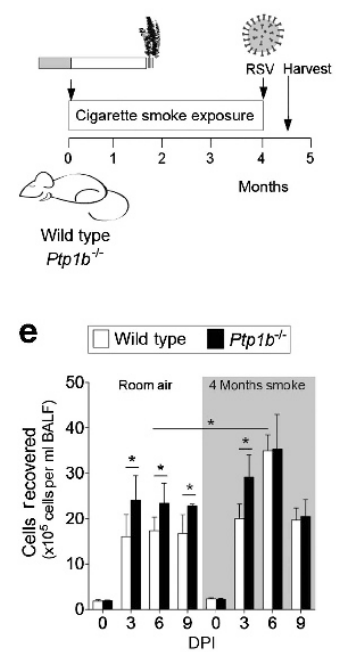
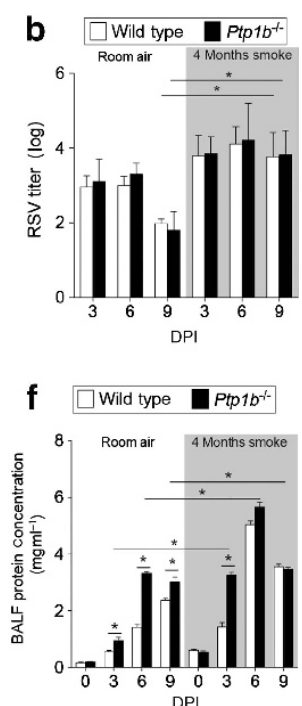
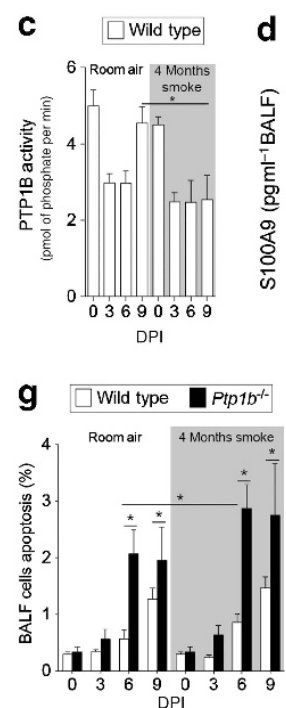
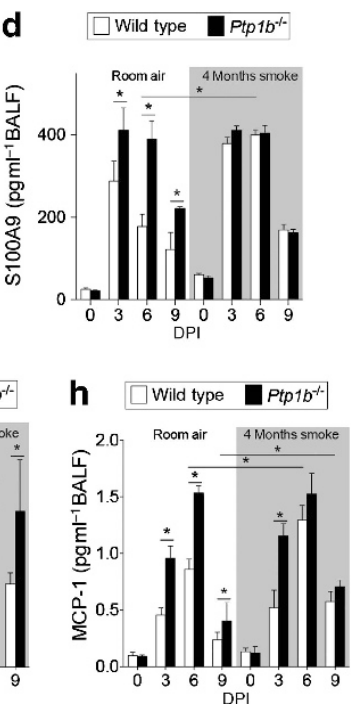

Figure 7 Cigarette smoke exposure enhances respiratory syncytial virus (RSV) infection-induced lung damage. (a) Wild-type and $P$ tp $1 b^{-/-}$mice were exposed to cigarette smoke for 4 months before intranasal infection with $1 \times 10^{6}$ plaque-forming units (PFUs) of RSV and animals were killed $0,3,6$, and 9 days post infection (DPI). (b) Lung RSV titer, (c) lung protein tyrosine phosphatase 1B (PTP1B) activity, (d) bronchoalveolar lavage fluid (BALF) levels of S100A9, (e) BALF cellularity, (f) BALF protein content, (g) BALF cells undergoing apoptosis, and (h) BALF monocyte chemoattractant protein-1 (MCP-1) levels were determined (white background $=$ room air and gray area $=$ smoke exposed for 4 months). Graphs are represented as mean \pm s.e.m., where each measurement was performed on 10 animals per group, with 6 replicates per animal. ${ }^{*} P<0.05$, comparing both treatments connected by a line.

enhanced apoptosis was still observed in $P t p 1 b^{-1-}$ animals compared with their wild-type littermates independent of their smoke exposure status (Figure 7g). Cigarette smoke and PTP1B both regulate other apoptosis-associated genes, in addition to S100A9. Thus, other factors outside of extracellular S100A9 may play a role in enhancing the apoptosis in $\mathrm{Ptp}_{\mathrm{p}} 1 \mathrm{~b}^{-1-}$ mice following smoke and RSV infection. Interestingly, MCP-1 secretion was also enhanced in smoke-exposed wild-type mice to levels observed in $P t p 1 b^{-1-}$ mice (Figure 7h). Therefore, cigarette smoke preconditions the lung for heightened S100A9 expression and aggravate viral exacerbations.

\section{Human BALF S100A9 levels have an inverse relationship with lung function}

S100A9 was measured in lung BALF from age-matched healthy control subjects, smokers, and subjects with COPD (Figure 8a). There is a significantly higher concentration of S100A9 in the BALF of COPD patients compared with healthy patients (Figure 8a). Importantly, S100A9 BALF levels have an inverse relationship with pulmonary function, determined by forced expiratory volume in 1 second $\left(\mathrm{FEV}_{1}\right)$ (percent predicted, $\mathrm{FEV}_{1} \%$ predicted is a ratio of the $\mathrm{FEV}_{1}$ of a patient over the $\mathrm{FEV}_{1} \%$ of the general population of similar size, sex, body composition) (Figure 8b). To determine whether cells from COPD lungs have an exaggerated S100A9 response during RSV infection, human bronchial epithelial cells isolated from healthy, smoking, and COPD donors were fully differentiated and cultured at the air liquid interface and were infected with RSV (Figure 9a). Cells from COPD subjects secreted more S100A9 (Figure 9b) and MCP-1 (Figure 9c) onto the apical surface of the cells (air exposed) than cells from healthy or smoking donors. A significant reduction in PTP1B activity was observed
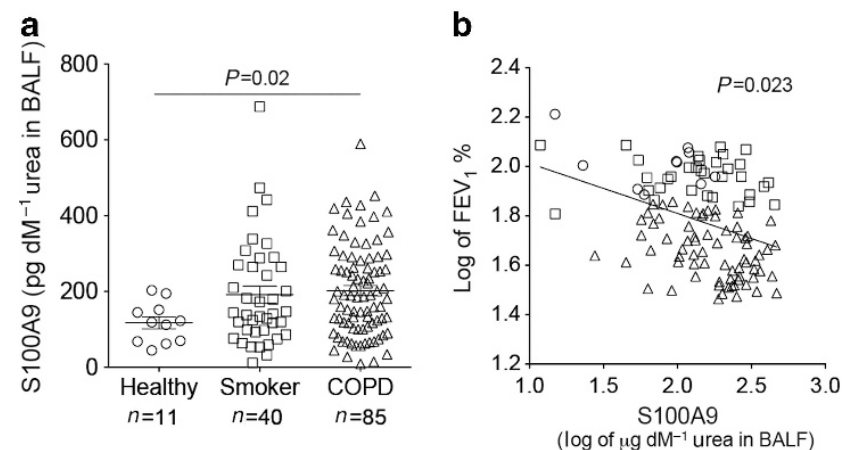

Figure 8 Extracellular S100A9 is increased in human chronic obstructive pulmonary disease (COPD) bronchoalveolar lavage fluid (BALF) samples. (a) S100A9 was measured in lung BALF from agematched healthy control subjects $(n=11)$, smokers $(n=40)$, and subjects with COPD $(n=85)$. BALF was standardized to urea levels. (b) BALF S100A9 levels had an inverse relationship with the $\mathrm{FEV}_{1} \%$ predicted ratio. Data are represented as log of mean \pm s.e.m., where each measurement was performed three times. $P$ values are shown, comparing both treatments connected by a line.

in the cells from COPD patients compared with cells from healthy and smoker donors (Figure 9d). Cells from COPD donors had a higher viral titer than cells from healthy smokers and nonsmokers (Figure 9e). To determine whether cells from COPD patients were more sensitive to S100A9 stimuli, S100A9 protein was added to the apical surface of cells at the air liquid interface (Figure 9f). The cells from COPD subjects had significant increases in MCP-1 and G-CSF secretion compared with the cells from other subject cohorts (Figure 9f). Elevated levels of lactate dehydrogenase were observed on the apical surface of COPD cells compared with healthy cells following S100A9 protein stimulation (Figure 9f). Therefore, enhanced 

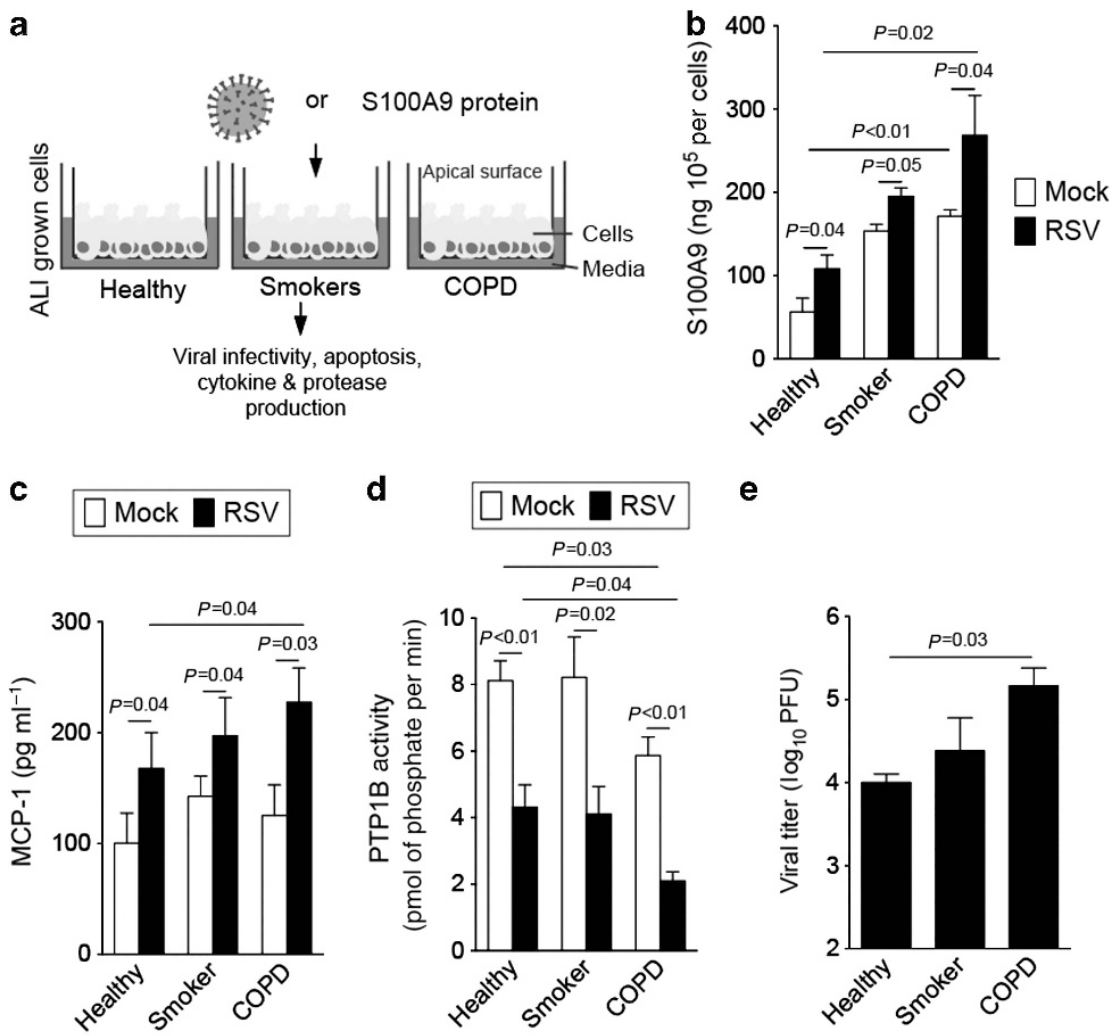

d

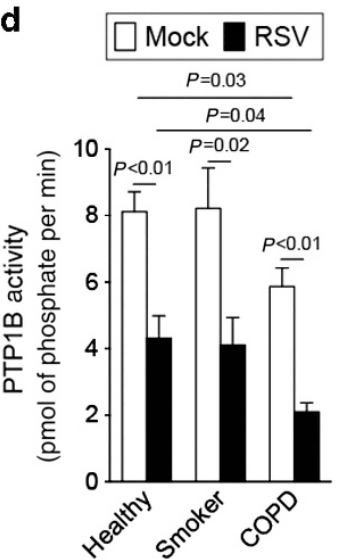

e
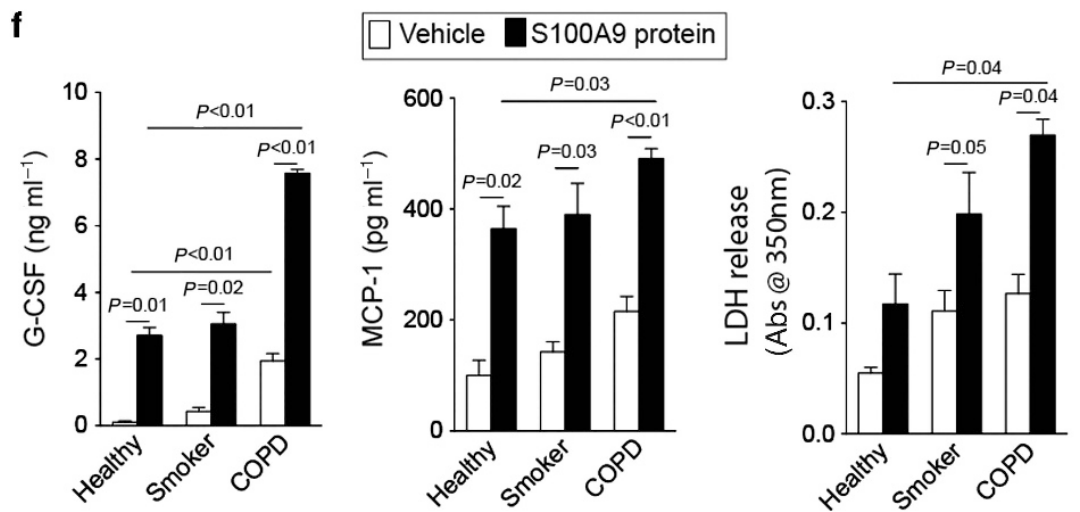

Figure 9 S100A9 signaling is heightened in airway epithelial cells from chronic obstructive pulmonary disease (COPD) patients. (a) Fully differentiated human airway epithelial cells grown at the air liquid interface (ALI) from healthy, smokers, and COPD individuals ( $n=4,3$, and 4 , respectively) were infected with (b-e) respiratory syncytial virus (RSV) or (f) treated with S100A9 protein for $2 \mathrm{~h}$ and washed with phosphate-buffered saline (PBS). Apical surface washes were taken $24 \mathrm{~h}$ later. RSV infection enhanced (b) S100A9 and (c) monocyte chemoattractant protein-1 (MCP-1) secretions. COPD cells had reduced (d) protein tyrosine phosphatase 1B (PTP1B) activity and (e) higher viral titer compared with cells from healthy and smoker donors. (f) Extracellular S100A9 stimuli increased MCP-1 and granulocyte colony-stimulating factor (G-CSF) secretion greater in COPD cells compared with cells from healthy and smoker donors. Cells from COPD donors also released greater lactate dehydrogenase (LDH) levels onto the apical surface of cells than the other groups. Graphs are represented as mean \pm s.e.m., where each measurement was performed 3 independent days on 3-4 donors per group. $P$ values are shown, comparing both treatments connected by a line. Abs, absorbance.

extracellular S100A9 in the lungs of COPD patients could exaggerate inflammatory mediators that contribute to the lung damage observed during RSV infection.

\section{TRIF signaling influences S100A9 expression}

Recently, TRIF signaling was shown to regulate S100A9 expression during influenza infection. ${ }^{17}$ To determine whether TRIF expression influences S100A9 expression during RSV infection, mouse bone marrow-derived macrophages from wild-type, Trif ${ }^{-1-}$, and $P t p 1 b^{-1-}$ animals were infected with RSV for $24 \mathrm{~h}$. Similar to our in vivo data, RSV infection resulted in S100A9 release that was enhanced in macrophages deficient for Ptp1b expression (Figure 10a). Loss of Trif expression reduced S100A9 release following RSV infection (Figure 10a). We further confirmed these data in human primary SAE cells using siRNA (Figure 10b and Supplementary Figure S2).

Treatment of SAE cells with S100A9 protein resulted in the phosphorylation of p38, extracellular signal-regulated kinase 

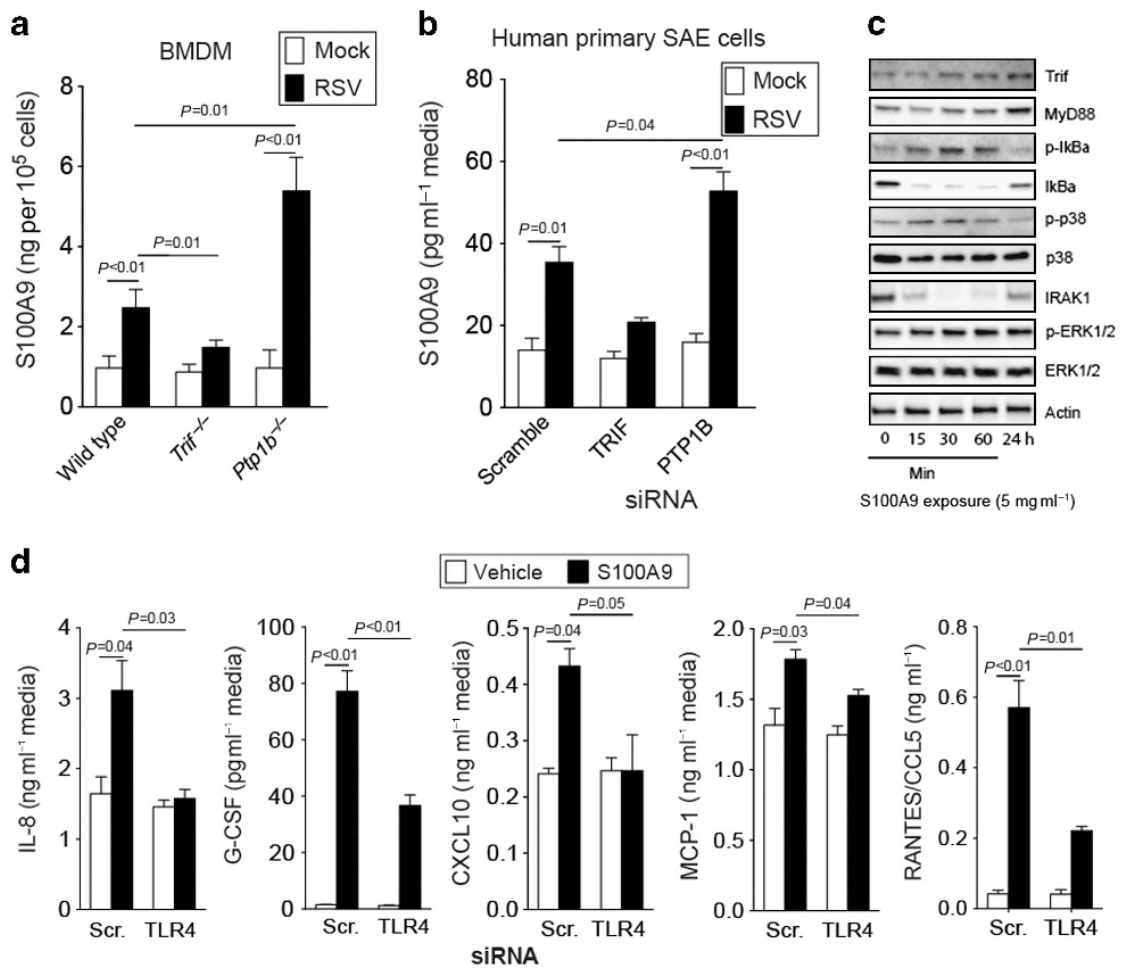

e

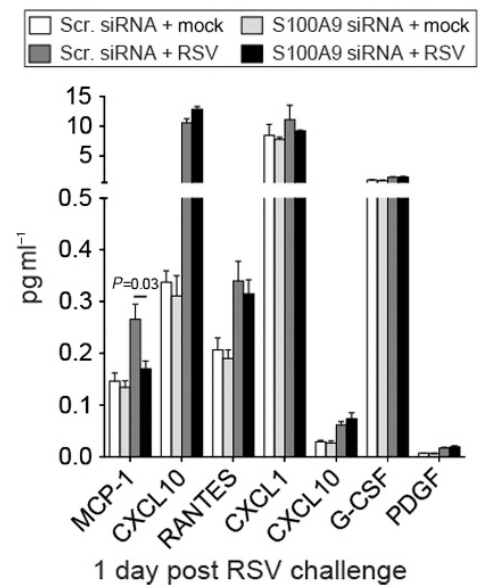

f

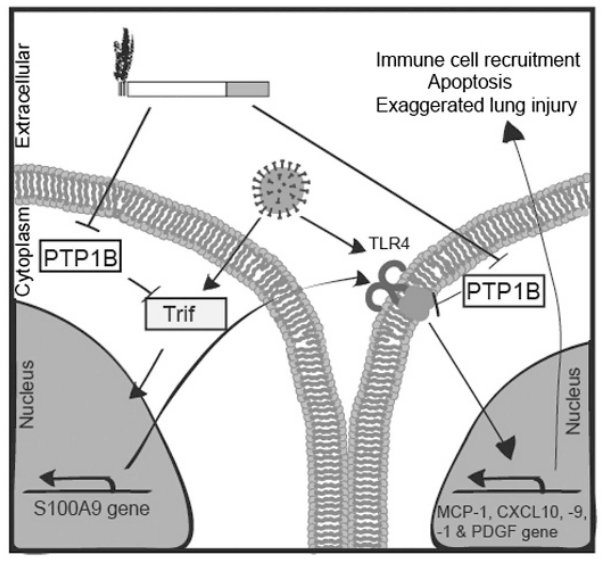

Figure 10 S100A9 induces Toll-like receptor (TLR)-mediated cytokine responses. (a) Primary bone marrow-derived macrophages (BMDMs) isolated from wild-type, Trif ${ }^{-1-}$, and Ptp $1 b^{-1-}$ mice were infected with respiratory syncytial virus (RSV) and enzyme-linked immunosorbent assay (ELISA) determined extracellular S100A9 protein levels, $24 \mathrm{~h}$ later. (b) Expressions of TRIF and PTP1B were silenced in human small airway epithelial (SAE) cells and cells were infected with RSV. ELISA determined extracellular S100A9 levels compared with cells transfected with scrambled (Scr.) small interfering RNA (siRNA). (c) SAE cells were treated with $5 \mu \mathrm{g} \mathrm{ml}^{-1} \mathrm{~S} 100 \mathrm{~A} 9$ protein for $0,15,30$, and $60 \mathrm{~min}$ and $24 \mathrm{~h}$. Trif, MyD88, I $\mathrm{kB} \alpha$, p38, ERK, and $\beta$-actin immunoblots were performed. Representative immunoblots demonstrate typical results from three separate assays. (d) S100A9 protein induced release of MCP-1, CXCL10, IL-8, RANTES, and G-CSF from SAE human cells in a TLR4-dependent manner confirmed in SAE cells transfected with either Scr. siRNA or siRNA specific for TLR4. (e) S100A9 expression was silenced in human SAE cells and cells were treated with RSV to determine the impact of RSV-induced S100A9 on cytokine expression. Graphs are represented as mean \pm s.e.m., where each measurement was performed three times on samples from three independent experiments. $P$ values are shown, comparing both treatments connected by a line. (f) Possible pathway for the regulation of S100A9-mediated lung damage during RSV-associated chronic obstructive pulmonary disease (COPD) exacerbations. CXCL10, chemokine (C-X-C motif) ligand 10; ERK, extracellular signal-regulated kinase; G-CSF, granulocyte colony-stimulating factor; IL-8, interleukin-8; MCP-1, monocyte chemoattractant protein-1; MyD88, myeloid differentiation primary response gene 88; PTP1B, protein tyrosine phosphatase 1B; RANTES, regulated on activation, normal T cell expressed and secreted; TRIF, TIR-domain-containing adapter-inducing interferon- $\beta$.

(ERK), and $\mathrm{I} \kappa \mathrm{B} \alpha$ and the induction of TRIF and MyD88 expression (Figure 10c), suggesting activation of TLR4 signaling. Therefore, we determined that extracellular S100A9 could induce cytokine secretion, via TLR4 signaling. SAE cells were transfected with TLR4 siRNA before S100A9 protein stimulation that demonstrated that TLR4 mediates S100A9induction of IL-8, G-CSF, CXCL10, MCP-1, and CCL5 (Figure 10d). To determine whether induction of S100A9 
expression during RSV infection was altering cytokine expression, S100A9 was silenced in SAE cells before RSV infection. Silencing S100A9 in human SAE cells reduced RSV infection-induced MCP-1 expression (Figure 10e). Therefore, we proposed that PTP1B might facilitate TRIF regulation of S100A9 expression that can contribute to TLR4-mediated inflammation (Figure 10f).

\section{DISCUSSION}

Respiratory viruses are a major cause of COPD exacerbations and these events play a critical role in disease progression and patient hospitalization. ${ }^{21}$ Thus, it is important to decipher how viral infections alter immune responses in the lung that can result in further loss of lung function in COPD patients. In this study, we demonstrated that PTP1B responses are desensitized in smoke-exposed lungs, leaving the lungs prone to enhanced S100A9 expression and consequent heightened lung damage during RSV infection. Ptp1b-deficient mice have enhanced immune cell recruitment to the lung, apoptosis, and cytokine production during RSV infection. Similar to influenza studies, ${ }^{17}$ RSV infection induces significant lung damage in a S100A9-dependent manner. Importantly, S100A9 responses are heightened in COPD lungs, with epithelial cells from COPD subjects exhibiting an intensified response to S100A9 protein compared with cells isolated from healthy donors. In addition, there is a negative relationship with BALF levels of S100A9 and lung function in patients. Extracellular S100A9 signaling is also critical in MCP-1 expression during RSV infection that could further affect immune cell infiltration. ${ }^{22}$ Overall, our study identifies that smoke-induced desensitization of PTP1B has the potential to enhance lung disease progression, in part, by augmenting the damaging effects of S100A9 expression in the lung during disease exacerbations caused by RSV. It is possible that a similar signaling cascade is used to promote loss of lung function in COPD subjects during infection with other viruses, such as rhinoviruses and influenza.

PTP1B undergoes activation following stimuli with inflammatory factors, such as tumor necrosis factor- $\alpha .^{23}$ Once activated, PTP1B acts as a common negative regulator of nuclear factor- $\mathrm{\kappa B}, \mathrm{AKT}$, and mitogen-activated protein kinase activities ${ }^{5}$ and can protect mice against injury. ${ }^{5}$ Before this study, little was known about how PTP1B could protect against lung inflammation and remodeling. The ability of PTP1B to regulate immune cell infiltration and cell expansion is vitally important. Ptplb-deficient mice have increased numbers of monocyte/macrophages in the spleen and the bone marrow, which have increased activation because of increased expression of CD80. ${ }^{5}$ PTP1B deficiency alters the immune responses to macrophages and apoptosis ${ }^{5}$ and increases susceptibility to smoke-induced COPD symptoms. ${ }^{6}$ In our study, we observed enhanced macrophage numbers in the BALF. The fate of other cell types are altered by PTP1B expression, with loss of PTP1B in B cells ${ }^{16}$ or its pharmacological inhibition ${ }^{24}$ enhancing AKT phosphorylation. In a mouse ovalbumin asthma model, loss of Ptp1b elevates the frequency of eosinophils and eosinophil progenitors compared with wild-type mice. ${ }^{25}$ In our RSV model, elevated eosinophil infiltration and $\mathrm{T}$ helper type 2 cytokines were observed in $P t p 1 b^{-1-}$ mice during RSV infection compared with wild-type mice. Inhibition of S100A9 subdued these responses. Therefore, regulation of S100A9 expression by PTP1B functions as a negative mediator of lung allergic responses. $\mathrm{S} 100$ proteins have also been associated with neutrophil activation. ${ }^{26}$ S100A8 and S100A9 directly bind to p67phox and p47phox, ${ }^{26}$ critical components of the NADPH complex, and potentate NADPH oxidase activation in neutrophils. PTP1B regulation of PAMPs is not restricted to TLR4 signaling, as loss of PTP1B heightens TLR2 (lipoteichoic acid) and TLR3 (polyinosinic:polycytidylic acid) signaling in macrophages. ${ }^{5}$ Therefore, PTP1B modulates PAMP and DAMP signaling and immune cell responses in several key cell types observed in COPD exacerbation.

Considering our data, we propose the signaling cascade outlined in Figure 10f, where smoke and RSV infection subdue PTP1B responses and thereby enhance PAMP and DAMP signaling. The exaggerated S100A9 response subsequently activates signaling cascades, ${ }^{19}$ such as TLR4, to enhance lung damage. Of note, PTP1B is oxidized in COPD lungs ${ }^{6}$ and increased S100A9 secretion is detection in the lungs of COPD subjects $^{27}$ that could contribute to enhance loss of lung function. Enhancing PTP1B levels in patients may not be the best means of treatment as PTP1B negatively regulates insulin signaling, ${ }^{28}$ and enhancing PTP1B could have several secondary systemic effects in the body. However, targeting specific PTP1B-dependent signaling, such as S100A9, may be feasible considering our S100A9 neutralizing data. Equally, PTP1B regulated IL-17 expression during RSV infection that has been associated with lung pathology in $\mathrm{COPD}^{29}$ and in RSV infections. ${ }^{30}$ S100A9 regulation of key cytokines has previously been identified ${ }^{17,31-33}$ but we observed that MCP-1 induction during an RSV infection is dependent on S100A9 expression. The exact role of MCP-1 during viral infections is limited. MCP-1 protects the lung in murine aspiration pneumonitis model ${ }^{34}$ but it enhances cigarette smoke-induced emphysema in mice. ${ }^{35}$ Induction of MCP-1 by S100A9 may be a recovery response to protect the lung from further S100A9-associated damage, as MCP-1 aids in closure of mechanical wounds ${ }^{36}$ and inhibiting MCP-1 signaling enhances lung injury during influenza infection. ${ }^{37}$ Equally, MCP-1-activated immune cells could alter apoptosis in the lungs. ${ }^{38}$ Identifying the role of MCP-1 in viral-associated COPD exacerbations will be a major future endeavor for our group.

Extracellular S100A9 upregulates apoptosis during RSV infection. However, following smoke exposure, Ptp $1 b^{-1-}$ mice still experience enhanced apoptosis that appears to be independent of S100A9 signaling. We identified several other PTP1B-regulated genes that can play a significant role in cell death and survival. Cell death, which is induced by S100A9, can intensify inflammation in the lungs ${ }^{39}$ and culminate in the exacerbation of the underlying lung disease. S100 proteins can induce cell death by various mechanisms ${ }^{17,31-33}$ and these processes could contribute to S100A9-mediated cell death in COPD lungs following a viral exacerbation. Intracellular 
S100A9 may be involved in negatively regulating antiviral response or aid in viral replication. ${ }^{17}$ However, we have not observed changes in viral replication between wild-type and $P t p 1 b^{-1-}$ mice, whereas we observed higher S100A9 expression in $P t p 1 b^{-1-}$ mice. We principally observe RSV infection in airway epithelial cells that are susceptible to S100A9 stimuli for cytokine production and apoptosis and can influence microenvironment changes in the lungs to enhance allergic responses. However, we cannot exclude the role of other cells types in disease progression. Recently, S100A9 was determined to be required for the maturation of TLR3 and signaling of TLR $3{ }^{40}$ In fact, S100A9 colocalizes with TLR3 to facilitate TLR3 localizing with its agonist. ${ }^{40}$ This S100A9 interaction with TLR3 mediates interferon- $\beta$ and IL-12 production. ${ }^{40}$ PTP1B can regulate downstream TLR3 signaling, such as TRIF and IRF3, that can regulate TLR3 signaling in addition to S100A9 expression. However, PTP1B can modulate other TLRs as maintaining PTP1B activity attenuates the development of LPS-mediated lung injury in mice. ${ }^{41}$ This may be partially dependent on the expression of S100A9 as both S100A9 and LPS induce TLR4 but each in a distinct manner. ${ }^{42}$ However, S100A9 protects against Klebsiella pneumoniae infection, as $S 100 a 9^{-1-}$ mice have enhanced bacterial load and lung damage compared with their wild-type littermates. ${ }^{43}$ Therefore, additional information is required on the antimicrobial potential of both intracellular and extracellular S100A9 to numerous pathogens and whether dysregulation of S100A9 contributes to lung remodeling and disease progression in other lung infections.

S100A9 expression is also regulated by STAT3 (signal transducer and activator of transcription 3) signaling in cancer cells. ${ }^{44}$ Interestingly, JAK2 (Janus kinase 2) and STAT3 are substrates of PTP1B, ${ }^{45}$ with PTP1B deficiency significantly enhancing the phosphorylation of JAK2 and STAT3 ${ }^{45}$ Phosphorylated STAT3 binds directly to the S100A9 promoter. ${ }^{46}$ S100A9 also inhibits the differentiation of dendritic cells and macrophages and induces accumulation of myeloidderived suppressor cells. ${ }^{47} \mathrm{Ptp} 1 b^{-1-}$ mice have an expansion of myeloid-derived suppressor cells that is linked to STAT3 expression. Thus, it is conceivable that the inactivation of PTP1B during RSV infection could affect the resolution of inflammation after infection by enhancing myeloid-derived suppressor cell infiltration in the lung. However, this response needs to be tightly controlled as the complete lack of PTP1B expression clearly accentuated lung damage in our RSV infection models. The full impact of unregulated S100A9 in COPD viral exacerbations and the influence of STAT3 signaling require further investigation.

In summary, we have identified that loss of lung PTP1B activity leads to enhanced lung remodeling in animal COPD and RSV infection models by enhancing extracellular S100A9 levels. S100A9 regulates inflammation processes during virus infection and S100A9 expression appears to be TRIF dependent during RSV infection. Future studies targeting PTP1B-dependent genes may lead to the development of measures to combat infection-associated exacerbations in COPD and other lung diseases.

\section{METHODS}

Virus and submerged cell culture. Human RSV strain A2 (VR-1540; ATCC, Manassas, VA) was used throughout study and was maintained as previously described. ${ }^{12}$ Monolayers of human SAE cells from healthy subjects (Lonza, Walkersville, MD) were cultured under submerged conditions. SAE cells were only used for experiments at passages 3-6 and at a confluency of $\sim 70 \%$. Cells were treated with RSV at a multiplicity of infection of 0.3 for $24 \mathrm{~h}$. Cells were also treated with mock control ${ }^{12}$ or $5 \mu \mathrm{g} \mathrm{ml}^{-1}$ S100A9 protein. Cells were transiently transfected with siRNA for TRIF, TLR4, S100A9, PTP1B, and scrambled control siRNA (QIAGEN, Valencia, CA) before RSV infection. See Supplementary Figure S2 for silencing efficiency of siRNA.

Animal models. Ptp1b (Ptpn1 gene) knockout $\left(^{-/-}\right)$mice (FVB.129S4(B6)-Ptpn1 $1^{\text {tm1Bbk} / M m j a x), ~ o n ~ a ~ F V B / N J ~ b a c k g r o u n d, ~}$ were purchased from the Mutant Mouse Resource Center at Jackson Laboratories (Bar Harbor, ME). All mice were maintained in a specific pathogen-free facility at Mount Sinai Roosevelt Hospital. The 8-weekold mice were used at the initiation point for all experiments and each experimental parameter had at least 10 animals per group/time point. Mice were exposed to cigarette smoke in a chamber (Teague Enterprises, Davis, CA) for $4 \mathrm{~h}$ a day, 5 days per week at a total particulate matter concentration of $80 \mathrm{mg} \mathrm{m}^{-3}$. The University of Kentucky reference research cigarettes 3R4F (Lexington, KY) were used to generate cigarette smoke. Smoke exposure was continued for 4 months after RSV infection or mock administered $24 \mathrm{~h}$ after the final day of smoke exposure. Mice were anesthetized by intraperitoneal injection of a mixture of ketamine and xylazine (Sigma Aldrich, St Louis, MO). Animals were intranasally administered $1 \times 10^{6}$ plaqueforming units of RSV or mock, as previously described. ${ }^{48}$ Mice were weighed daily and were killed on days $0,1,3,5,7$, and 9 after RSV or mock administration. BALF and tissues were collected for analysis. Total BALF protein was quantified by bicinchoninic acid assay (Thermo Fisher, Waltham, MA). The lungs underwent pressurefixation and morphometric analysis in accordance with our previously published protocol ${ }^{12}$ and in accordance with the ATS/ERS (American Thoracic Society/European Respiratory Society) issue statement on quantitative assessment of lung structure. ${ }^{49}$ Fixed sections $(6 \mu \mathrm{m})$ of paraffin-embedded lungs were hematoxylin and eosin stained and were scored independently from 0 to 9 for each section: ${ }^{12}$ peribronchiolitis, perivasculitis, and alveolitis for inflammatory cells surrounding a bronchiole, a blood vessel, or within alveolar spaces, respectively. The scoring was as follows: 0 , no inflammation; $1-3$, scant cells but not forming a defined layer; $4-6$, one to three layers of cells surrounding the vessel; 7-9, four or greater layers of cells surrounding the vessel or bronchial. Every vascular vessel and bronchus was measured on multiple lung lobes from three different depths of sectioned tissue. Slides were randomized, read blindly, and scored for each parameter. Another group of FVB/NJ and $P t p 1 b^{-1-}$ mice were intraperitoneally injected with $100 \mu \mathrm{g}$ of normal goat IgG (Santa Cruz Biotechnologies, Santa Cruz, CA) or a neutralizing goat polyclonal IgG targeting murine anti-S100A9 (Santa Cruz Biotechnologies) $2 \mathrm{~h}$ before RSV administration. This overall study was performed in strict accordance with the recommendations in the Guide for the Care and Use of Laboratory Animals of the National Institutes of Health and Institutional Animal Care and Use Committee guidelines.

Bone marrow-derived macrophages were obtained from mice (wild-type, Trif ${ }^{-1-}$, and $P t p 1 b^{-1-}$ ) by flushing mouse tibiae and femurs with ice-cold phosphate-buffered saline through a $70 \mu \mathrm{m}$-wide cell strainer and seeding cells at $37^{\circ} \mathrm{C}$ for $2 \mathrm{~h}$ in a humidified atmosphere with $5 \% \mathrm{CO}_{2}$ and then removing nonadherent cells. Cells were incubated in growth medium (minimum essential medium, $10 \%$ fetal bovine serum, $2 \mathrm{~mm}$ glutamine, $100 \mathrm{IU} \mathrm{ml}^{-1}$ of penicillin, $100 \mathrm{~g} \mathrm{ml}^{-1}$ of streptomycin, and $20 \mathrm{ng} \mathrm{ml}^{-1}$ macrophage colonystimulating factor) for 7 days before RSV infection. 
Lung viral titer. Infectious virus titers were determined by plaque assays. Lungs of infected mice were excised and homogenized using a mechanical homogenizer (Kinematica, Bohemia, NY). The viral titers in the homogenates were quantified by plaque assay on epithelial cells using methyl cellulose overlay media (R\&D Systems, Minneapolis, MN) and staining with $0.5 \mathrm{mg} \mathrm{ml}^{-1}$ thiazolyl blue tetrazolium bromide (MTT; Sigma Aldrich) solution in phosphate-buffered saline for $3 \mathrm{~h}$ at $37^{\circ} \mathrm{C}$. Paraffin-embedded lung samples were sectioned at $5 \mu \mathrm{m}$ and stained with polyclonal anti-RSV antibody that recognizes RSV antigen from RSV A and B isolates (Abcam, Cambridge, MA) and antibodies using an immunoperoxidase technique (Vectastain ABC Elite kit, Vector Laboratories, Burlingame, CA) to determine areas of lung infected.

Apoptosis measurements. BALF cells were analyzed for apoptosis utilizing the LIVE/DEAD cell viability assay from Life Technologies (Carlsbad, CA) on the Guava easyCyte flow cytometer from EMD Millipore (Temecula, CA). The apoptotic cells were expressed as a percentage of total BALF cells. BALF cells were further characterized in macrophage, neutrophil, eosinophil, and lymphocyte cell populations by flow cytometry.

Cytokine measurements. Cytokine gene expression from mouse whole-lung tissue or human cells was performed by qPCR using Taqman probes (Life Technologies/Applied Biosystems, Beverly, MA). Human and mouse cytokines were examined in human cell media and mouse BALF, respectively, using beads assays (Bio-Rad Magnetic Cytokine Bead Panels) with the BioRad Bio-Plex 200 system (Bio-Rad, Hercules, CA). Mouse and human S100A9 levels were determined using a bead assay and enzyme-linked immunosorbent assay (both from R\&D Systems), respectively. BALF was standardized for each assay to urea levels, as determined by a commercially available assay as instructed by the manufacturer (Abnova, Walnut, CA). S100A9 fluorescence levels were determined in lung sections from both genotypes at 5 DPI and each field of the microscope was quantified using ImageJ software (http://imagej.nih.gov/ij/docs/index.html). Goat polyclonal anti-S100A9 antibody (Santa Cruz Biotechnologies) was utilized to stain sections. All regions of the lung sections were utilized to avoid selection bias. Relative fluorescence intensity was determined ( $n=10$ animals per group).

Intracellular signaling. PTP1B activity was determined as previously described. ${ }^{35}$ Cells or tissue were lysed in radioimmunoprecipitation assay buffer, centrifuged at $13,000 \mathrm{~g}$ for $10 \mathrm{~min}$, and supernatants were collected. Immunoblots were conducted to determine levels of caspase-

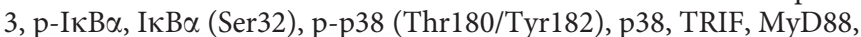
IRAK1, p-p44/42 mitogen-activated protein kinase (Erk1/2) (Thr202/ Tyr204), ERK1/2, $\beta$-actin (all antibodies from Cell Signaling Technologies, Beverly, MA) and S100A9 (Santa Cruz Biotechnologies).

Apoptosis array. The qPCR arrays were performed using the Bio-Rad PrimePCR apoptosis and survival panel (Bio-Rad; Apoptosis and Survival Tier 1-4 H384) to examine over 350 genes associated with cell survival and apoptosis. Data were analyzed and normalized with the Bio-Rad PrimePCR analysis software. Several PTP1Bregulated genes were validated by $\mathrm{qPCR}$, bead assays, and immunoblots.

Human samples. BALF was obtained from healthy never smokers, smokers, and COPD patients (see Supplementary Tables S1 and S2 for demographics). Written consent was obtained from all study participants and was approved by the institutional review board of the University of Miami. Human lungs were obtained from organ donors whose lungs were rejected for transplant. As some of these patients had a smoking history with macropathological evidence of emphysema, tissues from these donors were labeled as tissue from COPD patients. Consent for research was obtained by the Life Alliance Organ Recovery Agency of the University of Miami. All consents were approved by the institutional review board and conformed to the Declaration of Helsinki Principles.
Airway epithelial cells were isolated from healthy, smokers, and COPD lungs, dedifferentiated through expansion and redifferentiated at an air liquid interface on 24-mm T-clear filters (Costar Corning, Corning, NY) as previously described. ${ }^{50}$ RSV, mock, or S100A9 protein were added to the apical surface of the cultures and incubated for $2 \mathrm{~h}$ at $37^{\circ} \mathrm{C}, 5 \% \mathrm{CO}_{2}$. Subsequently, the apical surface was rinsed five times with phosphate-buffered saline and air liquid interface conditions were restored. After $24 \mathrm{~h}$, the apical surface was rinsed with $600 \mu \mathrm{l}$ phosphate-buffered saline and the rinse was harvested and investigated for cytokine production. The cells were collected for protein and RNA.

Statistical analyses. For statistical analysis, data from 10 animals or three separate cell experiments were pooled. Data are expressed as mean \pm s.e.m. Differences between groups of mice over time were compared by two-way analysis of variance. Individual differences between groups were tested by multiple comparison and analysis using the Bonferroni posttest analysis. Pairs of groups were compared by Student's $t$ test (two tailed). $P$ values for significance were set at 0.05 . All analyses were performed using GraphPad Prism Software (Version 5 for Mac OS X; La Jolla, CA).

SUPPLEMENTARY MATERIAL is linked to the online version of the paper at http://www.nature.com/mi

\section{ACKNOWLEDGMENTS}

This work was supported by grants made available to P.G. (Flight Attendant Medical Research Institute (YCSA 113380), R.F.F. (US National Institutes of Health 5R01HL098528-04), and M.A.S. (Flight Attendant Medical Research Institute 103027). We thank the James P. Mara Center for Lung Disease of the Pulmonary Division of Mount Sinai Roosevelt for their support and Gerard Turino and Charles Powell. We also thank the patients who participated in this study.

\section{DISCLOSURE}

The authors declared no conflict of interest.

c 2016 Society for Mucosal Immunology

\section{REFERENCES}

1. Elchebly, M. et al. Increased insulin sensitivity and obesity resistance in mice lacking the protein tyrosine phosphatase-1B gene. Science 283, 1544-1548 (1999).

2. Feldhammer, M., Uetani, N., Miranda-Saavedra, D. \& Tremblay, M.L. PTP1B: a simple enzyme for a complex world. Crit. Rev. Biochem. Mol. Biol. 48, 430-445 (2013).

3. Dube, N., Bourdeau, A., Heinonen, K.M., Cheng, A., Loy, A.L. \& Tremblay, M.L. Genetic ablation of protein tyrosine phosphatase $1 \mathrm{~B}$ accelerates lymphomagenesis of p53-null mice through the regulation of B-cell development. Cancer Res. 65, 10088-10095 (2005).

4. Berdnikovs, S., Abdala-Valencia, H. \& Cook-Mills, J.M. Endothelial cell PTP1B regulates leukocyte recruitment during allergic inflammation. Am. J. Physiol. Lung Cell. Mol. Physiol. 304, L240-L249 (2013).

5. Traves, P.G. et al. Pivotal role of protein tyrosine phosphatase 1B (PTP1B) in the macrophage response to pro-inflammatory and anti-inflammatory challenge. Cell Death Dis. 5, e1125 (2014).

6. Geraghty, P. et al. The glutathione peroxidase 1-protein tyrosine phosphatase 1B-protein phosphatase 2A axis. A key determinant of airway inflammation and alveolar destruction. Am. J. Respir. Cell Mol. Biol. 49, 721-730 (2013).

7. Miniño, A.M., Xu, J. \& Kochanek, K.D. Deaths: preliminary data for 2008. Natl. Vital Stat. Rep. 59, 1-72 (2010).

8. Miravitlles, M., Anzueto, A., Legnani, D., Forstmeier, L. \& Fargel, M. Patient's perception of exacerbations of COPD-the PERCEIVE study. Respir. Med. 101, 453-460 (2007).

9. Jain, S. et al. Community-acquired pneumonia requiring hospitalization among U.S. adults. N. Engl. J. Med. 373, 415-427 (2015). 
10. Walsh, E.E., Falsey, A.R. \& Hennessey, P.A. Respiratory syncytial and other virus infections in persons with chronic cardiopulmonary disease. Am. J. Respir. Crit. Care Med. 160, 791-795 (1999).

11. Zwaans, W.A., Mallia, P., van Winden, M.E. \& Rohde, G.G. The relevance of respiratory viral infections in the exacerbations of chronic obstructive pulmonary disease-a systematic review. J. Clin. Virol. 61, 181-188 (2014).

12. Foronjy, R.F., Dabo, A.J., Taggart, C.C., Weldon, S. \& Geraghty, P. Respiratory syncytial virus infections enhance cigarette smoke induced COPD in mice. PLoS One 9, e90567 (2014).

13. Phaybouth, V., Wang, S.Z., Hutt, J.A., McDonald, J.D., Harrod, K.S. \& Barrett, E.G. Cigarette smoke suppresses Th1 cytokine production and increases RSV expression in a neonatal model. Am. J. Physiol. Lung Cell. Mol. Physiol. 290, L222-L231 (2006).

14. Borchers, A.T., Chang, C., Gershwin, M.E. \& Gershwin, L.J. Respiratory syncytial virus-a comprehensive review. Clin. Rev. Allergy Immunol. 45, 331-379 (2013).

15. $\mathrm{Xu}, \mathrm{H}$. et al. Phosphatase PTP1B negatively regulates MyD88- and TRIFdependent proinflammatory cytokine and type I interferon production in TLR-triggered macrophages. Mol. Immunol. 45, 3545-3552 (2008).

16. Medgyesi, D. et al. The protein tyrosine phosphatase PTP1B is a negative regulator of $\mathrm{CD} 40$ and BAFF-R signaling and controls $\mathrm{B}$ cell autoimmunity. J. Exp. Med. 211, 427-440 (2014).

17. Tsai, S.Y. et al. DAMP molecule S100A9 acts as a molecular pattern to enhance inflammation during influenza $A$ virus infection: role of DDX21-TRIF-TLR4-MyD88 pathway. PLoS Pathog. 10, e1003848 (2014).

18. Kurt-Jones, E.A. et al. Pattern recognition receptors TLR4 and CD14 mediate response to respiratory syncytial virus. Nat. Immunol. 1, 398-401 (2000).

19. Vogl, T. et al. Mrp8 and Mrp14 are endogenous activators of Toll-like receptor 4, promoting lethal, endotoxin-induced shock. Nat. Med. 13, 1042-1049 (2007).

20. Pouwels, S.D. et al. Increased serum levels of LL37, HMGB1 and S100A9 during exacerbation in COPD patients. Eur. Respir. J. 45, 1482-1485 (2015).

21. Rohde, G. et al. Respiratory viruses in exacerbations of chronic obstructive pulmonary disease requiring hospitalisation: a case-control study. Thorax 58, 37-42 (2003).

22. Belperio, J.A. et al. Critical role for the chemokine MCP-1/CCR2 in the pathogenesis of bronchiolitis obliterans syndrome. J. Clin. Invest. 108, 547-556 (2001).

23. Zabolotny, J.M., Kim, Y.B., Welsh, L.A., Kershaw, E.E., Neel, B.G. \& Kahn, B.B. Protein-tyrosine phosphatase $1 \mathrm{~B}$ expression is induced by inflammation in vivo. J. Biol. Chem. 283, 14230-14241 (2008).

24. Xie, L. et al. Cellular effects of small molecule PTP1B inhibitors on insulin signaling. Biochemistry 42, 12792-12804 (2003).

25. Berdnikovs, S. et al. PTP1B deficiency exacerbates inflammation and accelerates leukocyte trafficking in vivo. J. Immunol. 188, 874-884 (2012).

26. Doussiere, J., Bouzidi, F. \& Vignais, P.V. The S100A8/A9 protein as a partner for the cytosolic factors of NADPH oxidase activation in neutrophils. Eur. J. Biochem. 269, 3246-3255 (2002).

27. Merkel, D., Rist, W., Seither, P., Weith, A. \& Lenter, M.C. Proteomic study of human bronchoalveolar lavage fluids from smokers with chronic obstructive pulmonary disease by combining surface-enhanced laser desorption/ ionization-mass spectrometry profiling with mass spectrometric protein identification. Proteomics 5, 2972-2980 (2005).

28. Seely, B.L. et al. Protein tyrosine phosphatase 1B interacts with the activated insulin receptor. Diabetes 45, 1379-1385 (1996).

29. Linden, A., Hoshino, H. \& Laan, M. Airway neutrophils and interleukin-17. Eur. Respir. J. 15, 973-977 (2000).

30. Newcomb, D.C. et al. IL-17A inhibits airway reactivity induced by respiratory syncytial virus infection during allergic airway inflammation. Thorax 68, 717-723 (2013).
31. Atallah, M. et al. Constitutive neutrophil apoptosis: regulation by cell concentration via S100 A8/9 and the MEK-ERK pathway. PLoS One 7, e29333 (2012).

32. Ghavami, S. et al. S100A8/A9 induces autophagy and apoptosis via ROSmediated cross-talk between mitochondria and lysosomes that involves BNIP3. Cell Res. 20, 314-331 (2010).

33. Li, C. et al. A novel p53 target gene, S100A9, induces p53-dependent cellular apoptosis and mediates the p53 apoptosis pathway. Biochem. J. 422, 363-372 (2009).

34. Raghavendran, K. et al. Acid and particulate-induced aspiration lung injury in mice: importance of MCP-1. Am. J. Physiol. Lung Cell. Mol. Physiol. 289, L134-L143 (2005).

35. Hautamaki, R.D., Kobayashi, D.K., Senior, R.M. \& Shapiro, S.D. Requirement for macrophage elastase for cigarette smoke-induced emphysema in mice. Science 277, 2002-2004 (1997).

36. Christensen, P.J., Du, M., Moore, B., Morris, S., Toews, G.B. \& Paine, R. 3rd Expression and functional implications of CCR2 expression on murine alveolar epithelial cells. Am. J. Physiol. Lung Cell. Mol. Physiol. 286, L68-L72 (2004).

37. Narasaraju, T., Ng, H.H., Phoon, M.C. \& Chow, V.T. MCP-1 antibody treatment enhances damage and impedes repair of the alveolar epithelium in influenza pneumonitis. Am. J. Respir. Cell Mol. Biol. 42, 732-743 (2010).

38. Yang, D., Elner, S.G., Chen, X., Field, M.G., Petty, H.R. \& Elner, V.M. MCP1 -activated monocytes induce apoptosis in human retinal pigment epithelium. Invest. Ophthalmol. Vis. Sci. 52, 6026-6034 (2011).

39. Bergsbaken, T., Fink, S.L. \& Cookson, B.T. Pyroptosis: host cell death and inflammation. Nat. Rev. Microbiol. 7, 99-109 (2009).

40. Tsai, S.Y. et al. Regulation of TLR3 Activation by S100A9. J. Immunol. 195, 4426-4437 (2015).

41. Grinnell, K.L., Chichger, H., Braza, J., Duong, H. \& Harrington, E.O. Protection against LPS-induced pulmonary edema through the attenuation of protein tyrosine phosphatase-1B oxidation. Am. J. Respir. Cell Mol. Biol. 46, 623-632 (2012).

42. Riva, M. et al. Induction of nuclear factor-kappaB responses by the $\$ 100 \mathrm{~A} 9$ protein is Toll-like receptor-4-dependent. Immunology 137, 172-182 (2012).

43. Achouiti, A. et al. Myeloid-related protein-14 contributes to protective immunity in gram-negative pneumonia derived sepsis. PLoS Pathog. 8 , e1002987 (2012).

44. Li, C., Zhang, F., Lin, M. \& Liu, J. Induction of S100A9 gene expression by cytokine oncostatin $M$ in breast cancer cells through the STAT3 signaling cascade. Breast Cancer Res. Treat. 87, 123-134 (2004).

45. Zhang, J. et al. Protein tyrosine phosphatase 1B deficiency ameliorates murine experimental colitis via the expansion of myeloid-derived suppressor cells. PLoS One 8, e70828 (2013).

46. Lee, M.J. et al. Interleukin-6 induces S100A9 expression in colonic epithelial cells through STAT3 activation in experimental ulcerative colitis. PLoS One 7, e38801 (2012).

47. Cheng, P. et al. Inhibition of dendritic cell differentiation and accumulation of myeloid-derived suppressor cells in cancer is regulated by S100A9 protein. J. Exp. Med. 205, 2235-2249 (2008).

48. Foronjy, R.F., Taggart, C.C., Dabo, A.J., Weldon, S., Cummins, N. \& Geraghty, P. Type-l interferons induce lung protease responses following respiratory syncytial virus infection via RIG-I-like receptors. Mucosal Immunol. 8, 161-175 (2015).

49. Hsia, C.C.W., Hyde, D.M., Ochs, M. \& Weibel, E.R. ATS/ERS Joint Task Force on the Quantitative Assessment of Lung Structure. An official research policy statement of the American Thoracic Society/European Respiratory Society: standards for quantitative assessment of lung structure. Am. J. Respir. Crit. Care Med. 181, 394-418 (2010).

50. Nlend, M.C., Bookman, R.J., Conner, G.E. \& Salathe, M. Regulator of G-protein signaling protein 2 modulates purinergic calcium and ciliary beat frequency responses in airway epithelia. Am. J. Respir. Cell Mol. Biol. 27, 436-445 (2002). 